Title: Post transplant liver graft schistosomiasis in a migrant from Sub-Saharan, Italy 2017

Running head: Post-transplant Schistosoma spp liver graft infection

Keywords: liver transplantation; Schistosoma; schistosomiasis; infection; outcome; patient; migrant; Italy; Europe

Authors: Paola Carrai MD ${ }^{1 *}$, Lorenzo Zammarchi, $\mathrm{MD}^{2,3^{*}}$, Luca Emanuele Pollina MD4, Luca Giordani ${ }^{5} \mathrm{MD}$, Valentina Mangano ${ }^{5} \mathrm{PhD}$, Riccardo lapoce $\mathrm{MD}^{6}$, , Francesca Rinaldi, MD², Alessandro Bartoloni, MD, DTM²,3 Franco Filipponi MD,PhD, Paolo De Simone MD,PhD ${ }^{1}$ Fabrizio Bruschi MD $5,7, \#$

\title{
Affiliations:
}

1. Hepatobiliary Surgery and Liver Transplantation Unit, University of Pisa Medical School Hospital, Pisa, Italy.

Emails: p.carrai@ao-pisa.toscana.it;

franco.filipponi@med.unipi.it;

paolo.desimone@unipi.it

Tel.: +39050995421

Fax: +39050995420

2. Department of Experimental and Clinical Medicine, University of Florence, Florence, Italy. Emails: lorenzo.zammarchi@unifi.it;

francesca.rinaldi.222@gmail.com,

alessandro.bartoloni@unifi.it;

Tel: + 390557949431

3. Referral Center for Tropical Diseases of Tuscany, Infectious and Tropical Diseases Unit, Careggi University Hospital, Florence, Italy.

4. Pathology, University of Pisa Medical School Hospital, Pisa, Italy.

Email: I.pollina@ao-pisa.toscana.it 
Tel: +39050995692

5. Department of Translational Research, N.T.M.S., University of Pisa, Via Roma 55, 56126 Pisa, Italy. Emails:

lucagiordani@hotmail.it

valentina.mangano@unipi.it

fabrizio.bruschi@med.unipi.it

6. Infectious Diseases, University of Pisa Medical School Hospital, Pisa, Italy.

\section{Email: r.iapoce@ao-pisa.toscana.it}

\section{Tel .+39050995431}

7. Programma Monitoraggio delle Malattie Parassitarie, AOU Pisana, Pisa, Italy.

* These authors contributed equally to this article

\# Corresponding author

\section{Biosketch}

Paola Carrai, MD is a liver transplant hepatologist. She graduated from the University of Pisa and completed her post-graduate residency program in Internal Medicine at the same University. During the last 15 years, Dr. Carrai has been serving as transplant hepatologist for pre- and posttransplant patients and has accumulated wide experience in the treatment of liver decompensation in waitlist patients, immunosuppressive therapy, and viral treatment of hepatitis $C$ and $B$ virus infection pre- and post-transplant.

\section{Abstract}

We report a case of post-transplant liver graft infection with Schistosoma spp in a migrant from Sub-Saharan Africa transplanted for HBV-related cirrhosis with pre-transplant undiagnosed schistosomiasis. The emergence of tropical diseases in non-endemic areas warrant screening protocols for donors and recipients with exposure in endemic areas.

\section{Text}


Schistosomiasis is a chronic parasitic disease affecting 230 million people worldwide [1]. The infection occurs largely in sub-Saharan Africa (SSA) with a prevalence between10 and $\geq 50 \%$ [2]. In non-endemic areas like Italy, prevalence rates between 7 and $34 \%$ are reported in migrants from SSA $[3,4]$. The infection is acquired when free-swimming cercariae penetrate the skin of people exposed to infested freshwater [1]. After penetrating the skin, the parasites migrate through the venous and general circulation, and finally concentrate in mesenteric or bladder veins, where they present in the form of male-female couples. Adult Schistosomas usually live up to 3-10 years, but parasites surviving for 40 years have been reported [1]. Adult females produce hundreds to thousands of eggs daily. These in turn may be eliminated with urine or feces, or become trapped in nearby tissues where they induce a local granulomatous inflammation and fibrosis leading to tissue damage [1]. SSA is endemic for four Schistosoma species. Schistosoma mansoni and $S$. haematobium are widespread in the African continent, whereas S. intercalatum and S. guineensis are less common and restricted to West and Central Africa [1, 5]. In the early phases, schistosomiasis may be completely asymptomatic, while hepatic, intestinal, and genito-urinary complications have been reported in chronic patients [2]. Herein we report a case of post transplant liver graft infection with Schistosoma spp in a patient from SSA.

\section{The study}

In July 2016, a 20-year-old male migrant from Liberia was admitted to our Unit for decompensated hepatitis B virus (HBV)-related cirrhosis. The patient had arrived in Italy 1 month earlier after a 3month stay in Libya and a 4-month stay in Algeria. His previous clinical history was unremarkable. On admission, his blood tests showed slight leucopenia without eosinophilia; thrombocytopenia (platelet 63,000/uL); total bilirubin 33.86 mg/dL; AST 99U/L; ALT 39 U/L; alkaline phosphatase 167 $\mathrm{U} / \mathrm{L}$; albumin $3 \mathrm{~g} / \mathrm{dL}$; INR 2.41, and serum creatinine $0.85 \mathrm{mg} / \mathrm{dL}$. His model for end-stage liver disease (MELD) score was 31. The viral serology was positive for HBV surface antigen (HBsAg), antibody to HBV core antigen (anti-HBc-lgG), and antibody to hepatitis delta virus (anti-HDV). HBV-DNA was $1.6 \mathrm{log}$. The abdominal CT scan showed marked splenomegaly $(26 \mathrm{~cm})$ and mild ascites. The esophagogastroduodenoscopy revealed F1 esophageal varices. 
Treatment with entecavir $0.5 \mathrm{mg}$ was started, and the patient underwent liver transplantation from an Italian deceased donor. The routine pre-transplant screening for infectious disease was unremarkable, showing previous EBV, CMV and Toxoplasma gondii infections. Histology of the native liver confirmed the diagnosis of HBV-related macronodular cirrhosis. Post-transplantation, the patient was started on quadruple immunosuppression with anti-CD25 (basiliximab, Simulect ${ }^{\mathrm{TM}}$, Novartis Italy, Origgio (VA)); tacrolimus (Prograf ${ }^{\mathrm{TM}}$, Astellas, Assago (MI)) adjusted to a target trough level of 8-10 ng/mL; mycophenolate mofetil $500 \mathrm{mg}$ b.i.d., and steroids from the anhepatic phase and tapered to $25 \mathrm{mg} /$ day from day 7 . Treatment with entecavir was continued and combined with anti-HBs immunoglobulin.

Two months after transplantation, the routine follow-up exams showed a 5-fold increase in transaminases without evidence of viral reactivation and partially responsive to increase of immunosuppression. Due to persistent increase in his liver function tests, the patient underwent liver biopsy 6 months post-transplantation. Histology showed inflammatory infiltration suggestive of mild acute cellular rejection and the presence of an oval structure with a terminal spine inside an eosinophilic granuloma (Figure 1), and consistent with Schistosoma spp egg. The serology for Schistosoma spp (Schisto II Western blot IgG, LDBIO Diagnostics, Lyon, France) and the urinary immunocromatographic test for circulating cathodic antigen (ICT-CCA, NADAL CCA Bilharzia test, Germany) tested positive, while parasitological examination of stool and urine (3 samples each) was negative.

Eight months post-transplantation, the patient was started on praziquantel $(40 \mathrm{mg} / \mathrm{kg} /$ day in two split doses on two separate days three weeks apart) and followed by ICT-CCA negativity and normal transaminases 2 weeks later. Strongyloides stercoralis Agar plate stool culture and antibodies were both negative. Retrospective examination of the recipient's native liver was negative for hepatic schistosomiasis, while the donor was born in Italy without history of travels in endemic areas for schistosomiasis. 


\section{Conclusions}

Recent migration from SSA is driving an increase in cases of reported schistosomiasis and other neglected tropical diseases in Southern Europe [4, 6], and evidence of local autochthonous transmission of Schistosoma in Europe is also available [7]. Along with other parasitic infections, such as Chagas disease and strongyloidiasis, schistosomiasis may have several implications for solid organ transplant patients. Hepatic schistosomiasis due to S. mansoni and S. japonicum may lead to progressive fibrosis and portal hypertension [8]. Even though the hepatocellular function is preserved until the late stage of the disease, may be necessary in the end-stage disease [8]. In a transplant center in Saudi Arabia, S. mansoni was indication to liver transplantation for $3 \%$ of patients undergoing liver transplantation [8]. In patients with S. haematobium infection, renal impairment is primarily caused by obstruction, reflux and stone formation related to fibrosis and calcification of tissue-trapped ova in the lower urinary tract [9]. Amyloidosis and glomerulonephritis may develop in S. haematobium or S. mansoni infection as a consequence of immune complexes containing worm antigens and deposited in the glomeruli [9]. In Egypt, schistosomiasis accounts for $10 \%$ of chronic renal failure and ranks second after diabetes mellitus [10]. If appropriately treated before transplantation, patients with schistosomiasis undergoing kidney or liver transplantation have outcomes comparable to other indications $[8,11]$. However, renal transplant recipients with schistosomiasis are reported to require higher dose of cyclosporine with subsequent higher incidence of nephrotoxicity, urinary tract infections and complications [11].

Experiences from endemic suggests that uncomplicated infection is not a contraindication to organ donation. In endemic areas, living donors should be screened for schistosomiasis and infected donors be treated with praziquantel before donation $[12,13]$. Transmission of living Schistosoma worms through the graft seems unlikely, since adult worms live in mesenteric and bladder veins, while granulomas containing eggs may be found in kidneys and liver [12]. This is why, due to praziquantel being active mainly against adult worms, the efficacy for patients receiving Schistosoma-infected grafts is largely debated [12]. Successful use of Schistosoma-infected liver grafts [12] has been reported, as well as kidney transplantation from donors with schistosomiasis [11]. 
The present case is consistent with graft infection from eggs produced by adult worms harbored by the recipient well before transplantation, rather than with donor-to-recipient transmission. The shape of the eggs was consistent with $S$. haematobium or its hybrid with other species (e.i. S. bovis, S. guineensis or S. curassoni based on the country of origin the shape and size of the terminal spine (M. Albonico, personal communication). The occurrence of schistosomiasis in nonendemic areas warrant implementation of screening protocols for donors and recipients with a history positive for exposure in endemic areas, including other parasitic disease such as strongyloidiasis [14] 


\section{References}

1. Othman A, El Ridi R. Schistosomiasis In: Helminth infections and their impact of Global Public Health. Edited by Bruschi F. Wien: Springer; 2014. pp. 49-92.

2. World Health Organization. Investing to overcome the global impact of neglected tropical diseases. Third who report on neglected tropical diseases. In. Geneva; 2015.

3. Martelli G, Di Girolamo C, Zammarchi L, Angheben A, Morandi M, Tais S, et al. Seroprevalence of five neglected parasitic diseases among immigrants accessing five infectious and tropical diseases units in Italy: a cross-sectional study. Clin Microbiol Infect 2017,23:335 e331-335 e335.

4. Beltrame A, Buonfrate D, Gobbi F, Angheben A, Marchese V, Monteiro GB, et al. The hidden epidemic of schistosomiasis in recent African immigrants and asylum seekers to Italy. Eur J Epidemiol 2017,32:733-735.

5. Mone H, Minguez S, Ibikounle M, Allienne JF, Massougbodji A, Mouahid G. Natural Interactions between S. haematobium and S. guineensis in the Republic of Benin. ScientificWorldJournal 2012,2012:793420.

6. Zammarchi L, Vellere I, Stella L, Bartalesi F, Strohmeyer M, Bartoloni A. Spectrum and burden of neglected tropical diseases observed in an infectious and tropical diseases unit in Florence, Italy (2000-2015). Intern Emerg Med 2017,12:467-477.

7. Beltrame A, Zammarchi L, Zuglian G, Gobbi F, Angheben A, Marchese V, et al. Schistosomiasis Screening of Travelers from Italy with Possible Exposure in Corsica, France. Emerg Infect Dis 2015,21:1887-1889.

8. El Moghazy W, Kashkoush S, O'Hali W, Abdallah K. Long-term outcome after liver transplantation for hepatic schistosomiasis: a single-center experience over 15 years. Liver Transp/ 2015,21:96-100.

9. Barsoum RS. Schistosomiasis and the kidney. Semin Nephrol 2003,23:34-41.

10. Essamie MA, Soliman A, Fayad TM, Barsoum S, Kjellstrand CM. Serious renal disease in Egypt. Int J Artif Organs 1995,18:254-260.

11. Mahmoud KM, Sobh MA, El-Agroudy AE, Mostafa FE, Baz ME, Shokeir AA, et al. Impact of schistosomiasis on patient and graft outcome after renal transplantation: 10 years' follow-up. Nephrol Dial Transplant 2001,16:2214-2221.

12. Vincenzi R, Neto JS, Fonseca EA, Pugliese V, Leite KR, Benavides MR, et al. Schistosoma mansoni infection in the liver graft: The impact on donor and recipient outcomes after transplantation. Liver Transp/ 2011,17:1299-1303.

13. Shokeir AA. Renal transplantation: the impact of schistosomiasis. BJU Int 2001,88:915-920.

14. Requena-Mendez A, Buonfrate D, Gomez-Junyent J, Zammarchi L, Bisoffi Z, Munoz J. EvidenceBased Guidelines for Screening and Management of Strongyloidiasis in Non-Endemic Countries. Am J Trop Med Hyg 2017,97:645-652. 
Figure 1: Liver biopsy six months after transplantation (HxE). Histology showed mild acute cellular rejection and an eosinophilic granuloma containing an ovum provided with a terminal spine, consistent with Schistosoma. A) Original magnification 200x, B) Original magnification 400x; C) Original magnification 1000x. 